\author{
F. Benedetti-Valentini \\ C. Villani \\ A. Laurito
}

M. Benedetti-Valentini

Received: 15 February 2006

Accepted: 12 September 2006

Published online: 18 December 2006

Presented at the Failure in Orthopaedics meeting, Rome, 27-29, October 2005.

\author{
F. Benedetti-Valentini $(\mathbb{\square}) \cdot$ A. Laurito \\ Department of Vascular Surgery \\ La Sapienza University \\ Rome, Italy \\ E-mail: chvs-rm@flashnet.it \\ C. Villani • M. Benedetti-Valentini \\ Department of Orthopaedics and \\ Traumatology \\ La Sapienza University \\ Rome, Italy
}

\section{Vascular complications of orthopaedic surgery: an update on diagnosis and management}

\begin{abstract}
Vascular complications in orthopaedic surgery are not frequent, but they can be severe and occasionally life-threatening. Present technologies make it easier to detect and successfully manage them, provided a high level of clinical suspicion is kept. Spontaneous complication is deep venous thrombosis (DVT), which occurs in $2.5 \%$ of patients undergoing hip or knee arthroplasty and can be treated by prophylactic doses of low molecular weight heparin (LMWH). D-Dimer blood test and duplex scanning are the pillars of diagnosis. LMWH (6000 U twice daily) is standard therapy but in selected cases thrombolysis or surgical venous thrombectomy can be used. Iatrogenic complications come from surgical manoeuvres and instruments; risk factors are preex-
\end{abstract}

isting atheromatous lesions and reinterventions, both orthopaedic and vascular. These lesions can cause bleeding, ischaemia, embolism, pseudoaneurysms or arteriovenous fistula, and are classified accordingly. Diagnosis is done by duplex scanning, computed tomography or angiography (particularly intraoperative angiography) and should be timely in order to allow the earliest possible management. This is crucial for a successful result, which may avoid damage to the patient and medicolegal problems. District-specific complications and treatment are reviewed.

Key words Vascular complications • Orthopaedic surgery $\cdot$ Treatment of complications

\section{Introduction}

Vascular complications in orthopaedic surgery are not frequent, but they can be severe and occasionally life-threatening. At the same time, vascular techniques are often less invasive and more effective and can achieve better results in management of these complications. In this paper, we review the diagnostic approach to vascular complications, the selective indications for treatment and the medicolegal implications.

Vascular complications in orthopaedic surgery can be distinguished into spontaneous or iatrogenic types.
Spontaneous complications are not directly dependent on the surgical procedure, and include deep vein thrombosis (DVT) due mainly to circulatory stasis and haematologic changes. Iatrogenic complications are those actively provoked by the surgical manoeuvres and by instruments and tools.

\section{Deep vein thrombosis}

Incidence of DVT varies according to different operations and districts and also depends on diagnostic means. When 
${ }^{125}$ I-fibrinogen scanning was used in patients without specific prophylactic measures, the incidence of DVT was $48 \%, 51 \%$ and $61 \%$ in those undergoing surgery for hip fracture, hip arthroplasty and knee arthroplasty, respectively [1]. However, the majority of DVT cases occurs in the calf veins, disappears rapidly and has little clinical impact. The current incidence of vascular complications in orthopaedic surgery patients subjected to low molecular weight heparin (LMWH) prophylaxis appears to be $2.5 \%$ for DVT, $1 \%$ for non-fatal pulmonary embolism (PE) and $0.1 \%-0.5 \%$ for fatal PE after hip or knee arthroplasty $[2,3]$.

Besides clinically assessing a patient's probability of having DVT, the diagnosis should rest mainly on D-dimer blood test and duplex scanning ultrasound investigations. A positive D-dimer test sets high suspicion even in clinically silent conditions; duplex scan is a reliable means to identify a floating thrombus (Fig. 1). It is also instrumental in a correct follow-up of operated patients.

Computed tomography (CT) and magnetic resonance venography (MRV) can be useful in dubious cases. Association of radioisotope phlebography and pulmonary scintigraphy performed as sequential steps of the same investigation can be extremely useful [4]. This is carried out by injection of $6 \mathrm{mCi} 99 \mathrm{mTc}$-albumin macroaggregates, and clearly shows the site and extent of venous thrombosis and the non-perfused areas of the lungs (Fig. 2).

Prophylaxis is mandatory in major orthopaedic surgery, and should be done with LMWH, which provides high protection and low bleeding risk [5]. A dosage of $4000 \mathrm{U} /$ day, to be started the day before surgery, is advised since it was sufficient and effective in our experience.

Once DVT has appeared, aggressive treatment is needed to stop thrombus spread and to prevent recurrence, PE and post-phlebitic syndrome. It is still debated whether intravenous unfractioned heparin or subcutaneous LMWH should be used. Some studies suggested that LMWH was at least as effective and safe as heparin and carried a significant advantage in the reduction of major bleeding and mortality. We employ LMWH (6000 U twice daily) in usual cases and intravenous heparin when venous or pulmonary thrombolysis in carried out. After orthopaedic surgery, thrombolysis should never be systemic, but it should be selective in the pulmonary veins or intrathrombus in the proximal veins. In few patients, venous thrombectomy may still be indicated, particularly in cases with a severe ischaemic component, either phlegmasia alba or phlegmasia coerulea dolens. Although long-term patency after venous thrombectomy is low, the short-term results are good as far as limb salvage and avoiding PE are concerned.

Inferior vena cava (IVC) filters are another option, both alone and associated with thrombolysis or venous thrombectomy. Indications for IVC filter placement, selection of the type of filter and when the filter should be a

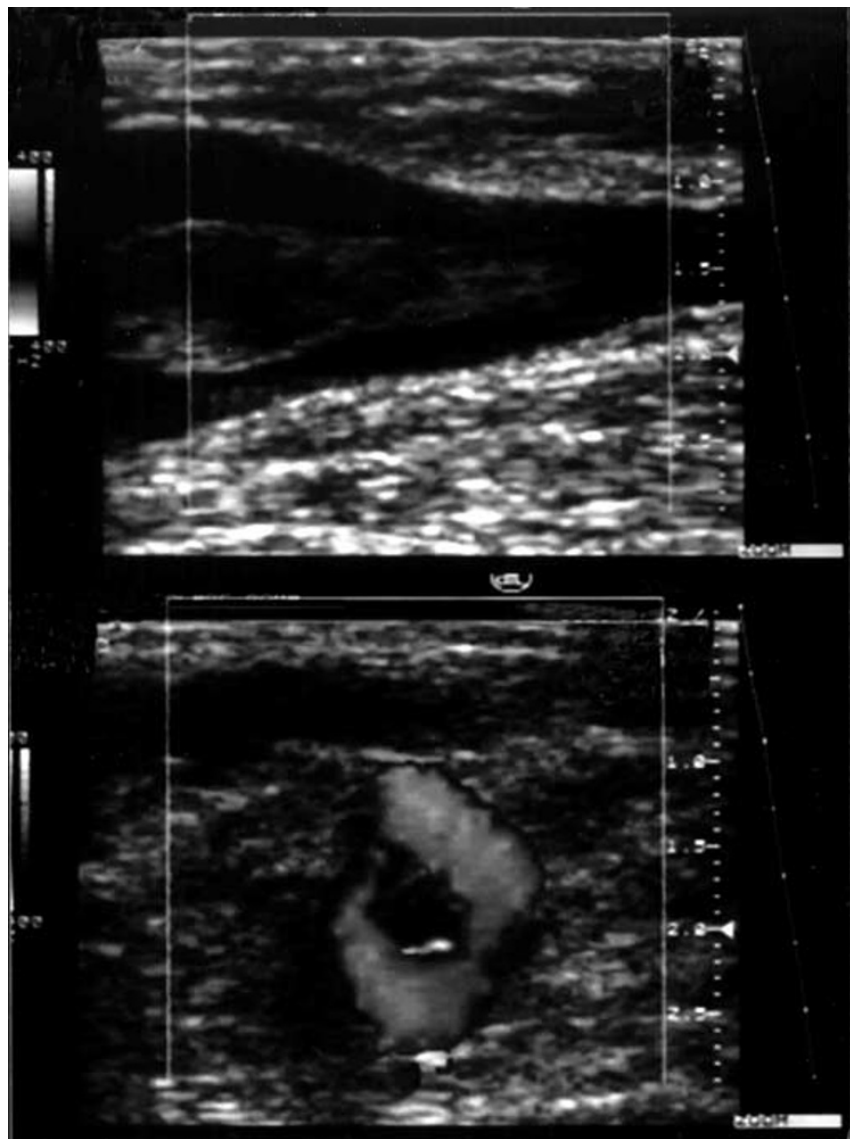

Fig. 1 Color-coded duplex scan showing a large thrombus partially floating inside the common femoral vein

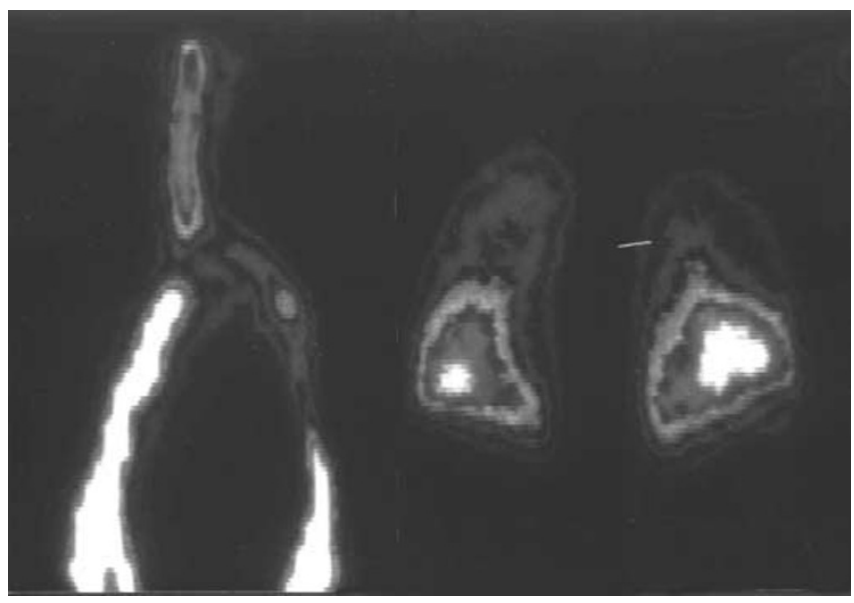

Fig. 2 Radioisotope (99m-Tc albumin macroaggregates) phlebography of the lower limbs associated with lung scintigraphy. A long filling defect is seen in the left iliac vein as are several areas of nonperfused lung tissue

temporary one were source of many arguments. The present orientation is that a filter should be placed in patients with proximal DVT and contraindications to or complications of anticoagulation, in recurrence of DVT after ade- 
quate anticoagulation, early after PE has occurred, when a floating thrombus is identified, and in recurrence of PE.

\section{latrogenic traumatic lesions}

Iatrogenic traumatic lesions may be classified according to a number of criteria, but the most useful method is probably a clinical classification (Table 1). Bleeding is one of the major features; it is usually life-threatening and can be an obvious external bleeding or a haematoma. Ischaemia may be severe or mild, posing diagnostic problems. Bleeding and ischaemia can be coupled in the same patient. In the medium or long-term, a pseudoaneurysm or an arteriovenous fistula may develop in patients surviving unrecognized or unrepaired vascular trauma. Embolism, both as macroembolism and microembolism with a blue toe syndrome, can result from manoeuvres on atheromatous arteries or on enlarged arteries containing thrombi. Thrombosis of the large veins can result from local trauma such as long compression, severe contusion, accidental tear or unsuccessful repairing efforts. Swelling of a limb may result from many causes such as compartment syndrome and venous stasis due to retraction.

Risk factors for iatrogenic vascular complications of orthopaedic surgery are associated with preexisting atherosclerotic disease and include extensive calcifications, soft atheroma, chronic occlusions and aneurysms. However, the highest risk [6] comes from reinterventions, vascular "redo" and, particularly, orthopaedic redo procedures. Scar tissue and changes in anatomic patterns account for many problems and complications.

Timely diagnosis is mandatory if the worst outcomes are to be avoided; however only $25 \%$ of vascular complications are identified during surgery, $50 \%$ are recognized during the first $24 \mathrm{~h}$ and the remainder are detected over days or months $[6,7]$. Such delays account for many negative outcomes and also for medicolegal problems and litigations. We surveyed 200 malpractice suits following iatrogenic vascular complications due to surgical and non-

Table 1 Iatrogenic vascular complications of orthopaedic surgery: clinical classification

\section{Bleeding}

Ischaemia

Bleeding and ischaemia

Embolism (macro or microembolism)

Pseudoaneurysm

Arteriovenous fistula

Post-trauma thrombosis (e.g. compression, contusion, tear) Swelling surgical procedures. The cases came from our experience as expert referees in judiciary courts and from the files of our hospital and follow-up of the related cases. Overall, surgery was involved in around $40 \%$ of cases (astonishingly less than non-surgical manoeuvres) but orthopaedic surgery was the cause in $25 \%$ of cases, and the main complaint was missed or delayed diagnosis leading to late treatment and poor results.

Diagnosis should always be based on clinical grounds and there should always be a high level of alert. When bleeding is severe, diagnosis is obvious; when bleeding is internal and a haematoma forms, diagnosis is difficult. Hypovolemic shock may ensue before the source is identified.

Similarly, when ischaemia is severe, it is seldom missed, but when the only sign is pulse disappearance or hypopulsatility, it is more likely to be overlooked. Blue toe syndrome due to multiple microemboli is sometimes wrongly interpreted as vasomotor response to trauma or as a result of arterial spasm and patchy vasoparalysis.

A pseudoaneurysm can present clearly as a pulsating mass in a palpable artery, but it is less obvious in deep arteries and in obese patients. An arteriovenous fistula can be easily diagnosed when it is large or superficial but diagnosis is difficult when it affects deep vessels. Severe oedema of one or both legs, usually with cyanosis, is sign of large or medium calibre vein involvement with thrombosis.

Neurologic signs and symptoms may ensue when a vertebral artery is affected, particularly in its intervertebral segment.

Laboratory investigations and angiography are instrumental in diagnosis and in planning the treatment. In a few cases, e.g. a large tear in the aorta or inferior vena cava, these tests cannot be used and the patient must be taken immediately to the operating room and swiftly operated upon. In most other cases, intraoperative duplex scanning or angiography may contribute a great deal. Doppler Current Wave (CW), digital oxygen saturation $\left(\mathrm{SaO}_{2}\right)$ and somatosensory evoked potentials (SSEP) are mainly used for monitoring during surgery and vessel retraction. Transcranial Doppler (TCD) should always be used when dealing with the vertebral artery directly or indirectly; a preoperative reading should be obtained in order to detect early changes during manoeuvres on the cervical spine. When there is time for additional investigations, CT with contrast medium and magnetic resonance imaging (MRI) are helpful.

Almost all surgical vascular or endovascular techniques can be and actually are used in different situations and areas of the body. Transcatheter embolization and stent graft placement are employed for small pseudoaneurysms or tears and even to stop bleeding as a first step before direct repair. Haemostasis can be obtained with clamps or with balloon catheters. The vessel wall can be repaired by 


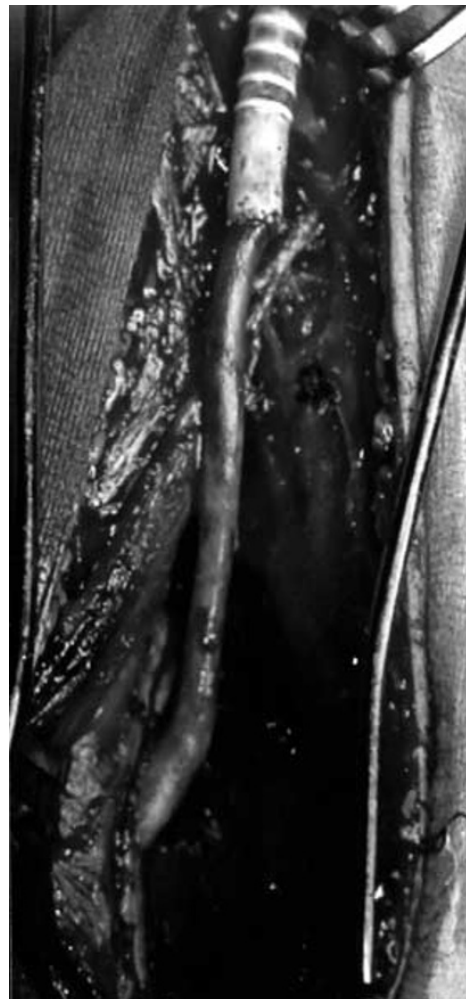

Fig. 3 Intraoperative image showing a composite graft, ePTFE plus autologous vein, used to reconstruct the posterior tibial artery

direct suture or by patching with various materials. Simple thrombectomy can be done, but it is seldom successful in achieving long-term patency since the vessel wall was itself a cause for thrombosis and remains injured. Graft interposition or bypass grafting are usually the most appropriate and most used techniques for severe or long lesions, both lacerations and occlusions. The best graft material is autologous vein, but for large arteries Dacron or expanded polytetrafluoroethylene (ePTFE) tubes may be more adequate. For distal vessels in selected cases, one can use composite graft, autologous vein plus ePTFE (Fig. 3).

\section{Procedure-specific complications}

There are particular problems for almost every district and type of orthopaedic operation.

Posterior C1-C2 transarticular screw fixation results in injuries to the vertebral artery in $1 \%-3 \%$ of cases and in neurologic deficit in 3\% [8]. Such injuries can lead to bleeding, occlusion, dissection, pseudo-aneurysm and arteriovenous fistula. Diagnosis is based on angiography and TCD assessment of the intracranial circulation. After tamponade, the best management is decided according to symptoms, technical possibilities and forecast of outcome. Vertebral artery ligation may be unavoidable although it leads to cerebral ischemia in $1.8 \%-3.1 \%$ of cases [9]. Stent placement and coil embolization are the right solution in selected cases [10]. Direct surgical repair, although demanding, is possible and often successful [11].

Abdominal aorta, inferior vena cava and iliac vessels are at risk in surgery of the lumbar spine. Anterior lumbar interbody fusion (ALIF), laminectomy and lumbar disc procedures are the most likely to lead to such injuries. Occasionally, the intestinal tract and the ureters are injured. The danger from disc surgery and laminectomy was first shown by De Saussure in 1959 when he identified 106 such cases in a questionnaire sent to 3000 orthopaedic surgeons; at that time the mortality rate was $47 \%$ [12]. The mechanism is usually slipping of a rongeur through the anterior longitudinal ligament during disc removal. A defect in that ligament may be a factor in some cases. At L1-L2 level, the abdominal aorta and the inferior vena cava are perforated; at L4-L5 or L5-S1 the aortic bifurcation and or the common iliac arteries and veins are involved. When both artery and vein are lacerated, if bleeding is tamponaded and the patient survives long enough, an arteriovenous fistula may result. Heart failure can then follow either, immediately or after many years [13].

Damage to a great vessel carries a mortality rate as high as $80 \%$ because of sudden blood loss and difficulties in repair. These orthopaedic procedures should never be undertaken in hospitals where a vascular surgeon is not readily available. In less severe situations, if the patient is haemodynamically stable, angiography or angio-CT are recommended in order to make the diagnosis and choose a strategy for treatment.

Conservative management is absolutely contraindicated even for small lesions because they often become acutely symptomatic and life- or limb-threatening. For the aorta and iliac arteries, direct suture is usually impossible and graft interposition is needed. This is successful in most cases, but graft infection has been reported [14] and demands an additional extra-anatomical bypass procedure. Great veins can be repaired by a simple running suture but sometimes, when part of the wall is damaged or "lost", a patch must be applied; first choice is an autologous venous panel (Fig. 4), since such tissue is less prone to infection and provides better long-term patency. Obviously, ureteral and bowel lesions should be looked for, identified if present and repaired during the same operation.

Thrombosis of the left common iliac artery is the typical vascular complication of anterior lumbar spine surgery, particularly ALIF. Retraction of this vessel is needed for technical reasons, but it can damage or overstrech a previously atherosclerotic artery particularly if it is prolonged overtime. Limb ischaemia ensues, sometime severe sometime mild, but failure to recognize it and to repair the artery can be harmful. Peripheral pulse monitoring or, much better, continuous or periodical ultrasound blood velocity 


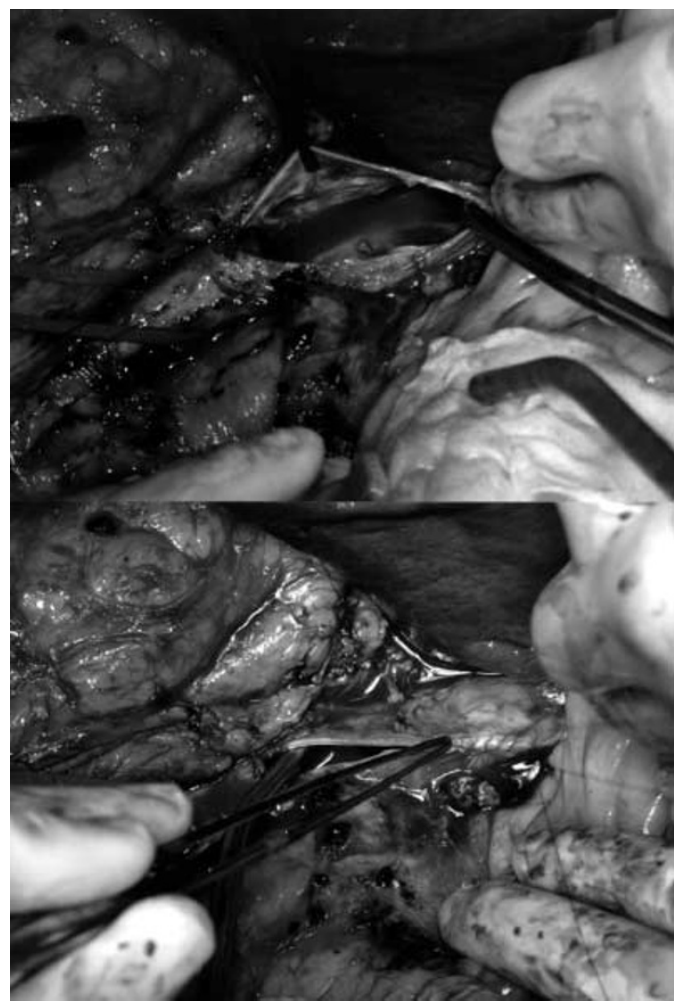

Fig. 4 Intraoperative image showing repair of the inferior vena cava by autologous venous patch

measurements can help to detect undue compression on the artery or thrombosis. SSEP monitoring and $\mathrm{SaO}_{2}$ measurements have also been employed for early detection of such complications [15]. Both methods were in agreement since loss of $\mathrm{SaO}_{2}$ was contemporary with loss of SSEP, suggesting also temporary nerve root ischaemia.

Prevention is the best approach to this complication starting from access. As a principle, the right retroperitoneal route should be reserved for L5-S1 procedures, while from L4-L5 upward a left retroperitoneal or a midline transperitoneal access should be preferred. We also suggest that a vascular surgeon be involved in the procedure in order to achieve better dissection of the vessels, which should be extensive. In order to achieve optimum mobilization of the common and external iliac artery and vein, the dissection should begin from the distal aorta and extend as distally as possible towards the femoral artery. While doing so, the left ureter is encountered, mobilized and spared.

When thrombosis has occurred, simple thrombectomy is seldom enough to provide long-lasting patency since one of the factors was damage to the arterial wall. Hence a graft is often needed to replace the artery or to bypass it: a Dacron tube is employed since, if the ureter and bowel were left intact, the chance of infection is minimal.

Percutaneous fixation of unstable pelvic ring fractures by insertion of an iliosacral lag screw carries a $1 \%-5 \%$ incidence of iatrogenic nerve root damage involving L5, S1 and the cauda equina [16]. It carries also a risk of vascular injuries to the iliac arteries and veins and their branches. The most specific is laceration of the superior gluteal artery with bleeding, pain and possible pseudoaneurysm formation.

The causes for this complication are generally surgeon inexperience and imaging difficulties at the time of screw placement. Anticoagulation after major trauma may also contribute to this potentially life-threatening complication. Diagnosis is made by CT or angiography, which in such cases becomes an interventional therapeutic procedure. The "endovascular way" should be first choice in order to avoid further surgical trauma to such already sick patients. Closure of the disrupted artery is achieved by gel foam and coil embolization.

Vascular complications of total hip arthroplasty (THA), acetabular revision and total knee arthroplasty (TKA) are few but severe and demand swift recognition and prompt surgical repair. Their incidence has been estimated as $<0.1 \%$ in THA and $<0.2 \%$ in TKA [6, 7]. However, they present with acute ischaemia in up to $60 \%$ of cases and arterial bleeding in around 20\%; diagnosis is delayed in $25 \%-40 \%$ of patients. External iliac artery and femoral artery and vein are at risk in THA but so are vessels in the pelvic region, particularly when screw fixation of acetabular components is used [17]. The popliteal artery and its branches are at risk in TKA, particularly when previous atherosclerotic lesions are present. In a total of 27 arterial injuries consequent to hip or knee surgery, the popliteal artery was involved in $12(44 \%)$ and the tibial artery in $5(18 \%)$. Diagnosis is made first by clinical means and then by duplex scanning and arteriography when needed. We suggest to use these imaging methods, particularly when facing problems with the popliteal artery because they help in planning surgical repair. As with the iliac artery, thrombectomy alone is seldom enough to guarantee patency (particularly with the politeal artery) and limb salvage. Dacron or ePTFE graft is used in iliac-femoral position; autologous saphenous vein, preferably from the contralateral limb, is better used in femoropopliteal or femorodistal revascularizations. Those procedures are highly successful when performed early after the complication has occurred. There is no place for conservative management in such cases.

Osteotomies in lower limb reconstruction, particularly limb lengthening, are a specific source of vascular complications involving mainly the superficial femoral artery, but also the femoral vein and branches of the popliteal artery. These vessels may be damaged by the drill, the osteotome or a fragment of bone, or be torn by continued distraction [18]. In upper tibial osteotomy, there is a higher risk of vascular injury, since the distal popliteal artery and the proxi- 
mal segment of its branches are less mobile and thus more vulnerable. Presentation is rarely as severe bleeding or acute ischaemia; in most cases the tear of the vessel wall is short, bleeding is tamponaded and a pseudoaneurysm follows. Pain, pulsating mass and swelling of the foot and leg or of the entire limb are the usual clinical picture. Diagnosis is made by laboratory investigations, mainly duplex scanning, and angiography; by these means an arteriovenous fistula can also be identified preoperatively. Repair can be done by endovascular means, either coil embolization or covered stents depending on the type of lesion and on the affected artery. In some cases, graft replacement or bypass is needed and autologous venous tissue is first choice. Outcome of the repair is almost regularly favourable in young individuals provided the diagnosis is timely, but in older patients a limb may be lost due to pre-existent arterial disease and loss of distal outflow.

\section{Conclusions}

Prior to any orthopaedic procedure, a careful and complete vascular assessment should be performed mainly to identify patients at high risk. Older patients with preexisting arterial or venous disease are more likely to run into vascular complications. Appropriate surgical technique, either open or endoscopic, is a top factor in avoiding these complications but, particularly in reinterventions, it may not suffice.

Collaboration with a vascular surgeon in anterior access to the spine is advisable since extensive vascular dissection is needed as well as careful vascular handling. Intraoperative monitoring should be employed during manoeuvres upon the cervical spine or the knee joint and in every anterior access to the spine.

Diagnosis of a vascular complication is based on clinical grounds and on a high level of alert. Intraoperative duplex scanning is sufficient in most cases to identify the problem and to provide the details needed for correction. Immediate vascular treatment, either open or endovascular, is mandatory if the best results are to be achieved. This must be firmly stated and clearly understood: there is no place for conservative treatment or delays in such cases. This also helps avoiding or controlling the medicolegal problems which almost regularly arise. Obtaining signed informed consent is obviously part of this strategy for defence of the surgeon.

\section{References}

1. Clagett GP, Anderson FA, Heit J (1995) Prevention of venous thromboembolism. Chest 108[Suppl 4]:312-318

2. Edelsberg J, Ollendorf D, Oster G (2001) Venous thromboembolism following major orthopaedic surgery: review of epidemiology and economics Am J Health Syst Pharm 58[Suppl 2]:4-13

3. Dahl OE, Caprini JA, Colwell CW, Frostick Sp, Haas S, Hull RD, Laporte S, Stein PD (2005) Fatal vascular outcomes following major orthopaedic surgery. Thromb Haemost 93:860-866

4. Massa R, Filippi L, Manini L, Fiore V (2001) Indagini radioisotopiche morfologiche e funzionali. In: SICVE (ed) Chirurgia vascolare. Minerva Medica, Turin, pp 57-65

5. Hull RD, Pineo GF (2000) Prevention and medical treatment of acute deep venous thrombosis. In: Rutherford $\mathrm{R}$ (ed) Vascular surgery. WB Sanders, Philadelphia, pp 1941-1958

6. Wilson JS, Miranda A, Johnson BL, Shames ML, Back MR, Bandyk DF (2003) Vascular injuries associated with elective orthopedic procedures. Ann Vasc Surg 17:641-644
7. Calligaro KD, Dougherty MJ, Ryan S, Booth RE (2003) Acute arterial complications associated with total hip and knee arthroplasty. J Vasc Surg 38:1170-1175

8. Wright NM, Lauryssen C (1998) Vertebral artery injury in C1-C2 transarticular screw fixation: results of a survey in the AANS/CNS section on disorders of the spine and peripher nerves. J Neurosurg 88:634-640

9. Islamoglu F, Posacioglu H, Memis A, Durmaz I (2002) Endovascular transcatheter occlusion for traumatic vertebral artery pseudoaneurysm. Eur J Vasc Endovasc Surg 20:576-578

10. Mendez JC, Gonzales-Llanos F (2005) Endovascular treatment of a vertebral artery pseudoaneurysm following posterior $\mathrm{C} 1-\mathrm{C} 2$ transarticular screw fixation. Cardiovasc Intervent Radiol 28:107-109

11. Berguer R (2000) Vertebrobasilar ischemia: indications, techniques, and results of surgical repair. In: Rutherford R (ed) Vascular surgery. WB Saunders, Philadelphia, pp 1823-1837
12. De Saussure RL (1959) Vascular injury coincident to disc surgery. J Neurosurg 16:222-228

13. Horton RE (1972) Arterial injuries complicating orthopaedic surgery. J Bone Joint Surg Br 54:323-327

14. Bingol H, Cingoz F, Yilmaz AT, Yasar M, Tatar H (2004) Vascular complications related to lumbar disc surgery. $\mathrm{J}$ Neurosurg 100[Suppl Spine 3]:249-253

15. Brau SA, Spoonamore MJ, Snyder L, Gilbert C, Rhonda G, Williams LA, Watkins RG (2003) Nerve monitoring changes related to iliac artery compression during anterior lumbar spine surgery. Spine J 3:351-355

16. Stephen DJG (1997) Pseudoaneurysm of the superior gluteal arterial system: an unusual cause of pain after a pelvic fracture. J Trauma 43:146-149

17. Fruhwirth J, Koch G, Ivanic GM, Seibert FJ, Tesch NP (1997) Vascular lesions in surquery of the hip joint. Unfallchirurg 100:119-123

18. Rickman M, Saleh M, Gaines PA, Eyres K (1999) Vascular complications of osteotomies in limb reconstruction. J Bone Joint Surg Br 81:890-892 